\title{
The FBI Laboratory Toolmark Topography Analysis Research for Objectively Qualifying the Degree of Similarity between Toolmarks
}

\author{
H.J. Seubert ${ }^{1}$ and E.D. Smith ${ }^{1}$ \\ ${ }^{1}$ Federal Bureau of Investigation Laboratory, Scientific Analysis Section, Firearms/Toolmarks Unit, \\ 2501 Investigation Parkway, Quantico, VA, USA
}

In 2009, a report by the National Academy of Sciences [1] called into question, amongst other issues, the lack of objectivity for visual toolmark identification (note that firearms identification is considered to be a subset of toolmark identification). The National Academies recommended development of objective toolmark identification criteria and error rate estimates. Currently in the forensic science community of toolmark identification, there is no objective standard for identification, no instruments capable to measure toolmark topographies (bullets and cartridge cases) coupled with an algorithm that can statistically analyze toolmark similarities for identification.

In 2012, the Firearms/Toolmarks Unit (FTU) of the FBI Laboratory began acquiring 3D instrumentation to determine whether this technology was feasible to enhance the forensic pattern based discipline of the firearms/toolmarks identification by providing objective information to establish a statistical significance. If successful, not only would these technologies provide objective data to support examiner conclusions, but possibly create higher efficiency for forensic casework analysis. Since that time, the FTU has acquired the following instruments for evaluation: Sensofar-Confocal Interferometry and Focus Variation; Cadre Forensics-Topmatch GS 3D; Alicona-Infinite Focus; EvoFinder; and GigaMacro. To assess these platforms, the FTU developed a validation plan, which encompassed a two-phase approach. Phase one included developing a method to assess the instruments measuring capabilities determining the collection parameters to acquire the image, and determine the appropriate sample data sets for collection and evaluation. The initial data samples that were selected for evaluation included previously distributed proficiency test that the FTU had and three test sets assembled from the FBI Laboratory's consecutively manufactured slides and barrels collection. The outcome of these data sets demonstrated 3D technology would be a viable operation for performing virtual comparisons on ammunition components and the FTU began its final step of the validation process, which included previously analyzed casework samples. The first system to have this last step finalized was the Cadre Forensics-Topmatch GS 3D. This presentation will discuss the implementation process, challenges encountered, proposed work flow and incorporation into operational casework.

\section{References:}

[1] National Research Council, Strengthening Forensic Science in the United States: A Path Forward, National Academy Press: Washington, D.C. (2009) (http://www.nap.edu/catalog/12589.html). 

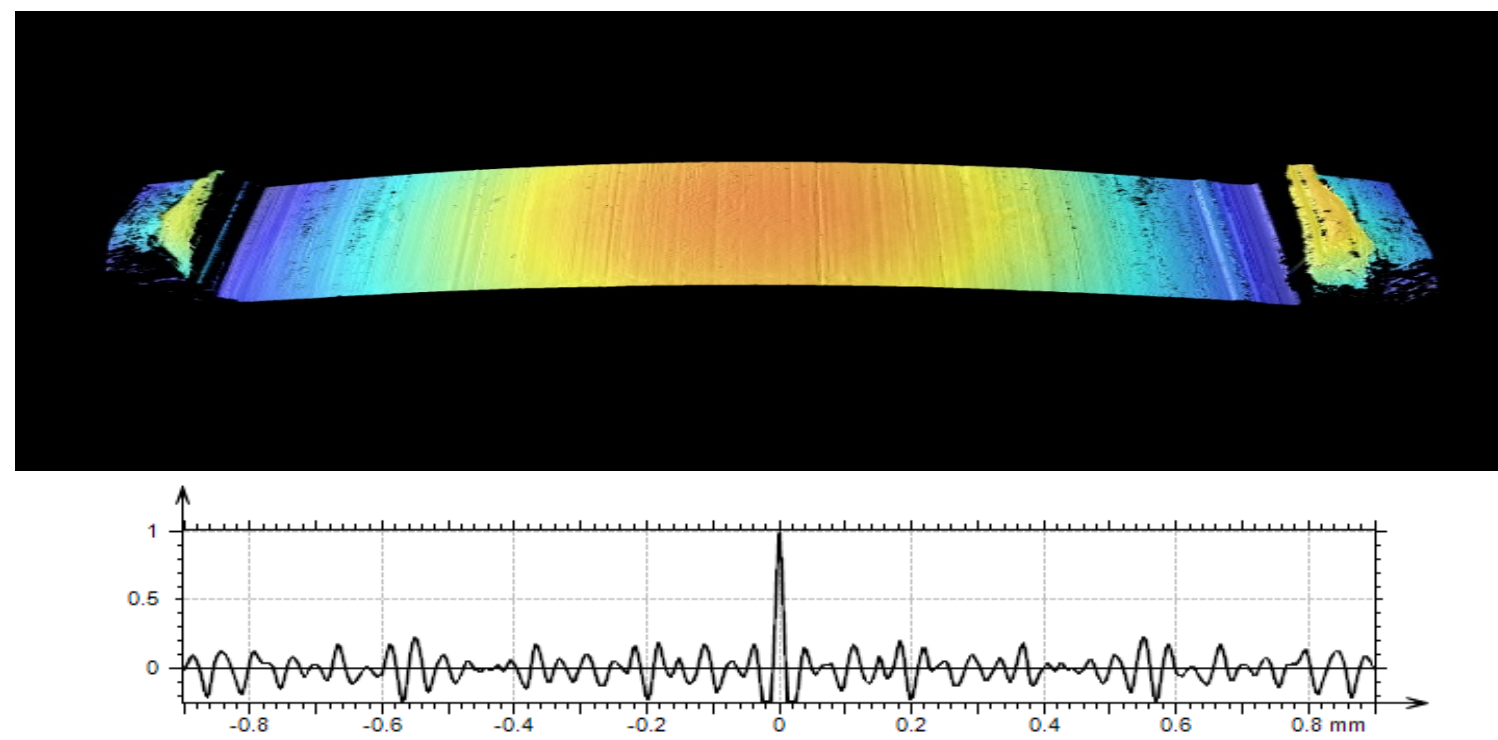

Figure 1. Scan of a land impression from a bullet scanned with the Sensofar Confocal Microscope and the cross correlation function showing the ouput for a match
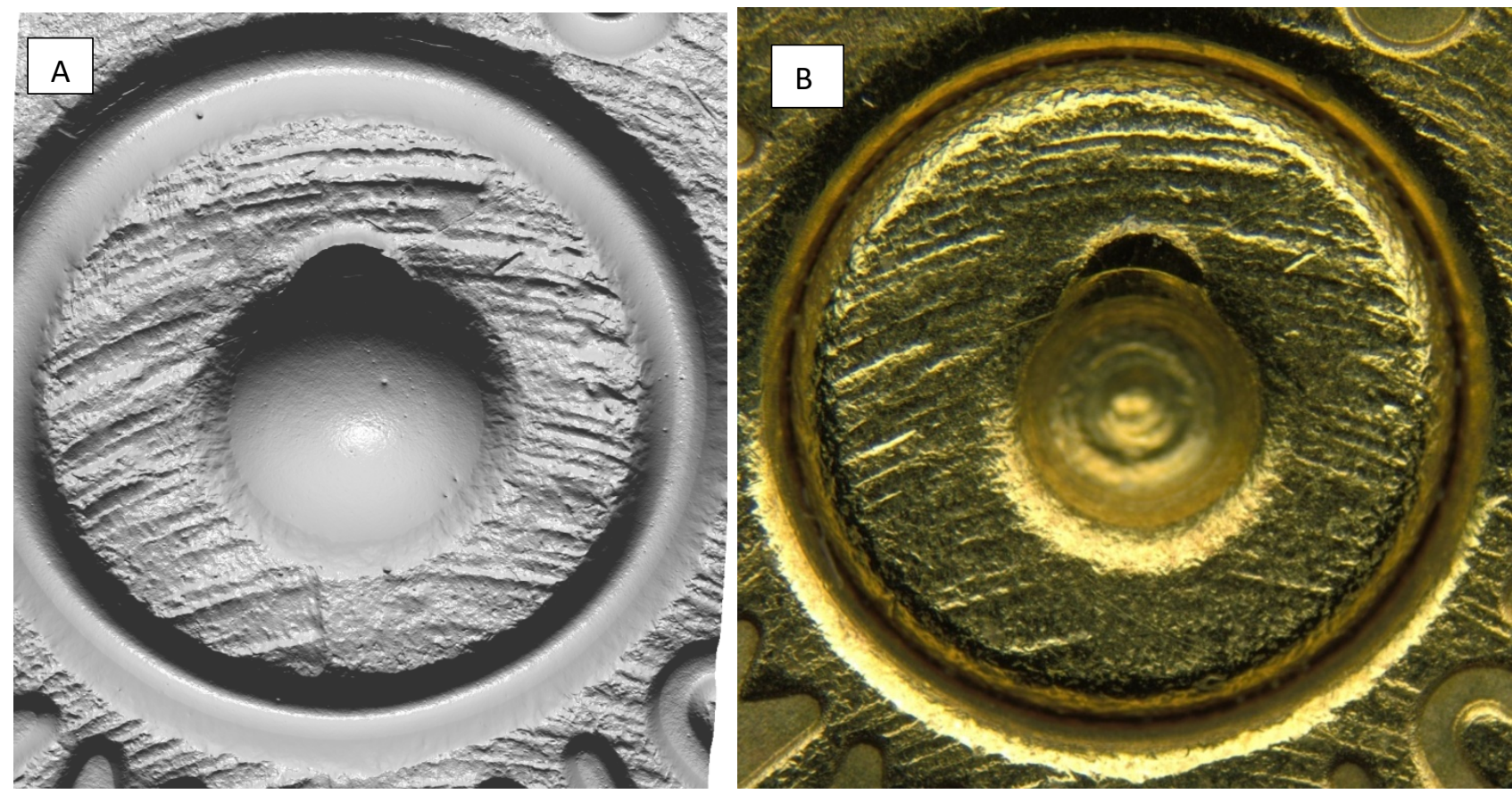

Figure 2. Image of a cartidge case from Cadre Gelsight (A) and an image of a cartidge case from Light Comparison Microscopy (B) 